\title{
Comparison of 3D-CRT and IMRT techniques in radiotherapy for post-prostatectomy patients with a higher risk of nodal involvement
}

\author{
Maria Poncyljusz, Paweł Kukołowicz, Joanna Chorąży, Beata Czyżew, Anna Jankowska, \\ Anna Paciorkiewicz, Piotr Pęczkowski, Małgorzata Pilichowska, Anna Zawadzka
}

Background. Irradiation of a larger volume of the target may lead to an increase of the doses delivered to the surrounding organs at risk (OAR) for post-prostatectomy patients with a higher risk of nodal involvement. It was anticipated that IMRT significantly improved OAR sparing. The aim of this study was to provide a dosimetric comparison between conformal radiotherapy (3D-CRT) and intensity-modulated radiotherapy (IMRT) treatment plans for patients with prostate cancer irradiated to the prostate bed and pelvic lymph-nodal area.

Materials and methods. The 3D-CRT and IMRT plans were created for ten patients after prostatectomy. The treatment plans were generated for the prostate bed (PTV1) and the pelvic lymph nodes (PTV2). The sum of PTV1 and PTV2 was irradiated to a mean dose of 46 Gy in 23 fractions, and additionally PTV1 was irradiated to a mean dose of $18 \mathrm{~Gy}$ in 9 fractions. Target coverage and the doses delivered to the pelvic bones, the rectum, the bladder, the bowel bag, and the femurs, were compared between techniques. The Wilcoxon signed-rank test was used to compare the dosimetric parameters.

Results. The dosimetric quality of 3D-CRT and IMRT plans were comparable for target coverage (the mean value of PTV1 V95\%, the mean value of PTV2 V95\% all > 99\%). The IMRT plans resulted in significant reductions in the pelvic bones V30[\%], V40[\%], the rectum V40[\%], V50[\%], V60[\%], the bladder V40[\%], V50[\%], V60[\%], the bowel bag V45[cc] and the femurs $\mathrm{V} 40[\%]$.

Conclusions. The analysis presented in this paper demonstrates that the IMRT technique reduces the delivered dose to the OARs. Most interesting was the possibility of reducing the delivered dose to the pelvic bones and the bowel bag. This allowed us to expect a decreased risk of acute hematologic toxicity and acute gastrointestinal toxicity.

NOWOTWORY J Oncol 2016; 66, 6: 440-444

Key words: postoperative radiotherapy, prostate cancer, intensity modulated radiotherapy, hematologic toxicity

\section{Background}

Intensity modulated radiotherapy (IMRT) has become a standard among modern treatment techniques. There are many publications which demonstrate the benefits of IMRT in radiotherapy for patients with prostate cancer [1-9]. However, only a few of them have evaluated the feasibility of this technique for patients after prostatectomy [10-12]. These studies show that IMRT, together with a helical tomotherapy (HT), allow for better local tumour control and spare organs at risk (OARs).
For post-prostatectomy patients with a higher risk of nodal involvement irradiation of the pelvic lymph nodes may improve the treatment outcome by potentially eradicating nodal micrometastases [13].

On the other hand, irradiation of a larger volume of the target may lead to an increase of the doses delivered to surrounding OARs, including the rectum, the bladder, the bowel bag, the femurs, and the pelvic bones. Clinical investigation conducted by Alongi et al. [14] showed that IMRT and HT significantly reduce the risk of acute genito-urinary 
and gastrointestinal toxicity when compared to conformal techniques (3D-CRT). However, in this paper, the dosimetric parameters of dose distribution have not been considered and the dose delivered to the pelvic bones was not taken into account.

The dose to the pelvic bones is a surrogate of the dose delivered to the bone marrow, which is an important factor in the treatment process. It is anticipated that IMRT significantly improves OAR sparing, including the pelvic bones, while maintaining a similar level of the target coverage when compared to 3D-CRT for the irradiation of the prostate bed and the pelvic lymph nodes.

The aim of this study was to compare the quality of the plans prepared with IMRT and 3D-CRT techniques in terms of the dosimetric parameters of dose distribution.

\section{Materials and methods}

Treatment plans for 10 patients treated between 2012 and 2014 were analysed.

The clinical indications for radiotherapy were as follows:

- tumour stage: pT3a / b - exceeding the prostate and/ /or infiltration of the seminal vesicle;

- positive surgical margin (R1);

- lymph node involvement $\mathrm{N}(+)$;

- PSA $>0.2 \mathrm{ng} / \mathrm{ml}$ at least 5 weeks after surgery.

The patients were given a pharmaceutical treatment 7 days prior to a planning CT scan, in order to prevent bowel distention. The patients were asked to drink $500 \mathrm{ml}$ of water 30 minutes prior to the CT scan and then before each irradiation session. The patients were scanned using a contrast computed tomography ( $3 \mathrm{~mm}$ slice thickness) in the supine position with a knee support and with their hands resting on their chest. Planning CT scans were obtained from the mid-level L4 up to $5 \mathrm{~cm}$ below the ischial tuberosities.

CTV1 included the prostate bed depending on the diagnosis with or without the seminal vesicles (classification
T3a - covered base of seminal vesicle, classification T3b - covered $2 / 3$ of seminal vesicle). PTV1 was created by extending the CTV1 by a $1 \mathrm{~cm}$ margin.

CTV2 included the pelvic lymph nodes and was drawn starting from the L5/S1 level up to the upper edge of the symphysis pubis. The external iliac nodes were drawn up to the top of the femoral heads. CTV2 was then grown isotropically by $7 \mathrm{~mm}$ to create PTV2.

The following at risk organs were contoured: the rectum, the bladder, the right and left femurs (femur L/R), the bowel bag, the pelvic bones. The left and right femurs, the bowel bag, the rectum and the bladder were drawn according to the recommendations of the RTOG pelvis atlas [15]. The bowel bag was cropped from PTV1 and PTV2 with no margin. The pelvic bones were delineated from the level of the ischial tuberosities up to the tops of the upper iliac, including the femurs.

The radiotherapy was delivered with a mean dose to PTV1 of 64 Gy and with a mean dose to PTV2 of $46 \mathrm{~Gy}$. The treatment was delivered in two phases. The prescribed dose of $46 \mathrm{~Gy}$ with a fractionation regime of $2 \mathrm{~Gy}$ per fraction was initially delivered to PTV1 and PTV2. During the second part, PTV1 was irradiated to $18 \mathrm{~Gy}$ in 9 fractions.

Individual 3D-CRT and IMRT treatment plans were designed for each patient using a commercial treatment planning system (Eclipse ver. 6.5, Varian Medical Systems, Inc.). The calculations were performed using the AAA algorithm (Anisotropic Analytical Algorithm, Ver. 10.0.28). The 3D-CRT plans were created according to our clinical protocol. The three field technique was used with $15 \mathrm{MV}$ photon beams and gantry angles of $0^{\circ}, 90^{\circ}, 270^{\circ}$. Appropriate AP wedges were applied to the lateral beams and the anterior beam was split to two separate beams as shown in Figure 1.

Sliding window IMRT plans were created using 5 photon beams with an energy of $6 \mathrm{MV}$ (gantry angles of $0^{\circ}$, $72^{\circ}, 144^{\circ}, 216^{\circ}, 288^{\circ}$ ). A small modification to the gantry angle was allowed in order to spare the femoral heads.
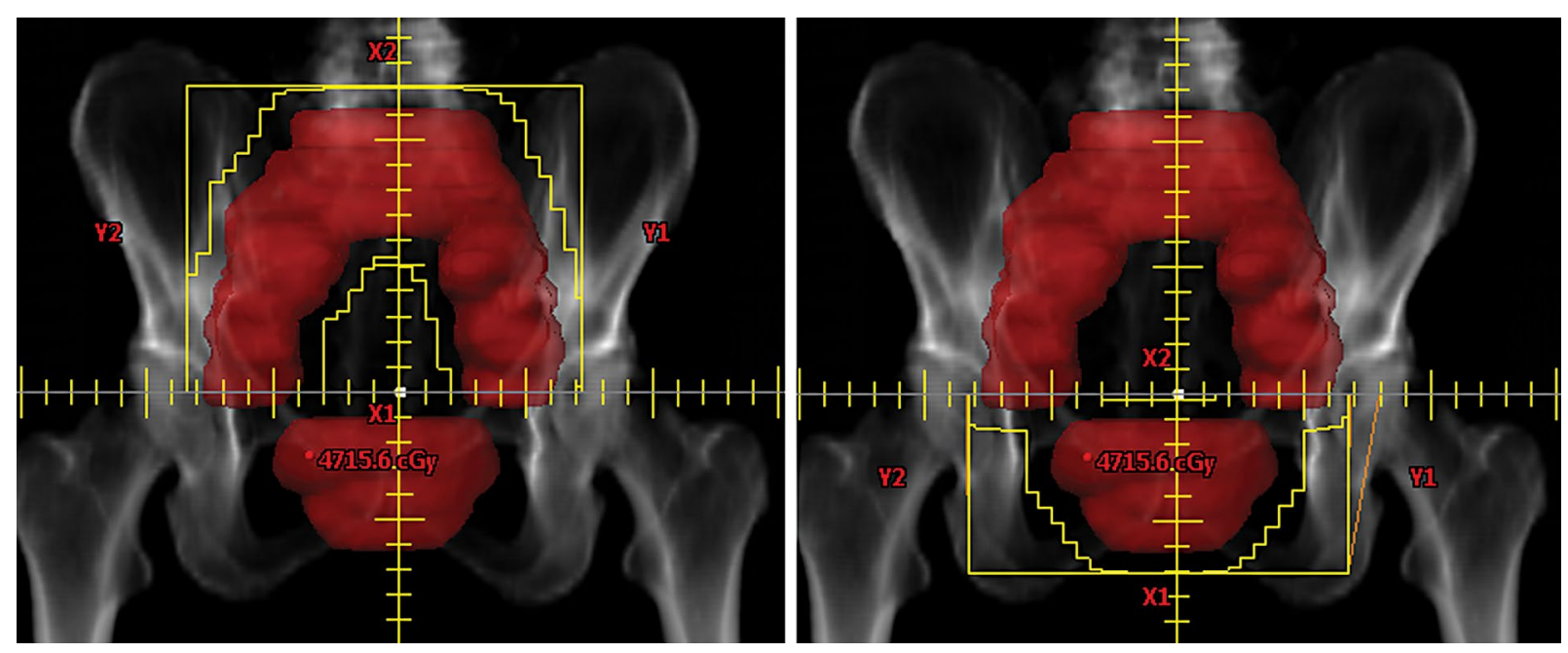

Figure 1. Field shapes for beams in the first stage of treatment. Gantry angle $0^{\circ}$. PTV1 and PTV2 are shown in red 
Table I. The dose distribution indices used for comparison

\begin{tabular}{lc}
\hline OAR & Analysed value \\
\hline Femur L and femur R & $\mathrm{V}_{40 G y}[\%]$ \\
Pelvic bones & $\mathrm{V}_{30 G y}[\%], \mathrm{V}_{40 G y}[\%]$ \\
Bladder & $\mathrm{V}_{40 G \mathrm{~Gy}}[\%], \mathrm{V}_{50 G y}[\%], \mathrm{V}_{60 G y}[\%]$ \\
Rectum & $\mathrm{V}_{40 G y}[\%], \mathrm{V}_{50 G y}[\%], \mathrm{V}_{60 G y}[\%]$ \\
Bowel bag & $\mathrm{V}_{45 G y}[\mathrm{ccc}]$ \\
\hline
\end{tabular}

A collimator angle of $3^{\circ}$ was chosen to minimise the overlap of interleaf leakage.

The PTV planning objective was to deliver more than $95 \%$ of the prescribed dose to a minimum of $98 \%$ of the target volume. The dose volume histograms were evaluated for both techniques for the PTV and for the organs at risk alternatively. Using the recommendations of the Quantitative Analysis of Normal Tissue Effects in the Clinic study (QANTEC) [16], the indices listed in Table I obtained for the 3D-CRT and the IMRT techniques were compared. The Wilcoxon signed-rank test was used and the $p$-values were calculated (Tab. I).

\section{Results}

According to this study, the mean value of PTV1 V95\% and the mean value of PTV2 V95\% for the 3D-CRT and the IMRT plans were comparable. The average volume of PTV1 receiving at least $95 \%$ of the prescribed dose was $99.2 \%$ and $99.7 \%$ for the 3D-CRT and the IMRT techniques, respectively. The average volume of PTV2, receiving at least $95 \%$ of the prescribed dose, was $99.0 \%$ and $99.9 \%$ for the 3D-CRT and the IMRT techniques, respectively. The average minimum doses delivered to the prostate bed were $59.9 \mathrm{~Gy}$ and $59.2 \mathrm{~Gy}$ for the 3D-CRT and the IMRT, respectively. The average prostate bed maximum doses were $65.2 \mathrm{~Gy}$ and $66.2 \mathrm{~Gy}$ for the 3D-CRT and the IMRT techniques, respectively. The lymph nodes received a patient-average minimum dose of 42.5 Gy and $42.4 \mathrm{~Gy}$ and a patient average maximum dose of $62.3 \mathrm{~Gy}$ and $61.8 \mathrm{~Gy}$ for the 3D-CRT and the IMRT, respectively. All these differences were not statistically significant (p-value $>0.05$ ).

The IMRT plans resulted in a significant reduction of doses delivered to the OARs (Table II). Due to anatomical differences between patients which cause large differences in the volumes of the bowel bag receiving a dose $45 \mathrm{~Gy}$ or higher, a patient-average value V45[cc] was not calculated and not compared in this study (Tab. II).

The average cumulative dose-volume histograms illustrating the differences between both techniques are shown in Figure 2. Due to the symmetry in patient anatomy, only results for the left femur were presented.
Table II. The average differences between values Vxx obtained using 3D-CRT and IMRT techniques, $\mathrm{p}$-values are based on the Wilcoxon signedrank test. Vxx is the volume receiving a dose of $x x G y$ or higher

\begin{tabular}{|c|c|c|c|}
\hline OAR & $V x x$ & p-value & $\begin{array}{l}\text { Average difference } \\
\text { Vxx }_{3 \mathrm{D}-\mathrm{CRT}}-\mathrm{Vxx}_{\text {IMRT }}\end{array}$ \\
\hline Femur L & $\mathrm{V}_{40 \mathrm{~Gy}}[\%]$ & 0.007 & 23.13 \\
\hline Femur $\mathrm{R}$ & $\mathrm{V}_{40 \mathrm{~Gy}}[\%]$ & 0.005 & 27.74 \\
\hline \multirow{3}{*}{ Pelvic bones } & $\mathrm{V}_{30 \mathrm{~Gy}}[\%]$ & 0.007 & 9.183 \\
\hline & $\mathrm{V}_{40 \mathrm{~Gy}}[\%]$ & 0.005 & 8.022 \\
\hline & $\mathrm{V}_{40 \mathrm{~Gy}}[\%]$ & 0.005 & 8.738 \\
\hline \multirow[t]{3}{*}{ Bladder } & $\mathrm{V}_{50 \mathrm{~Gy}}[\%]$ & 0.007 & 6.821 \\
\hline & $\mathrm{V}_{60 \mathrm{~Gy}}[\%]$ & 0.007 & 4.088 \\
\hline & $\mathrm{V}_{40 \mathrm{~Gy}}[\%]$ & 0.028 & 6.277 \\
\hline \multirow[t]{2}{*}{ Rectum } & $\mathrm{V}_{50 \mathrm{~Gy}}[\%]$ & 0.005 & 8.002 \\
\hline & $\mathrm{V}_{60 \mathrm{~Gy}}[\%]$ & 0.005 & 4.604 \\
\hline Bowel bag & $V_{40 G y}[c c]$ & 0.005 & - \\
\hline
\end{tabular}

\section{Discussion}

The dose distribution in PTV1 and PTV2 were similar in 3DCRT and IMRT plans. DVH analysis and the Wilcoxon test for the femurs $\mathrm{V}_{40 \mathrm{~Gy}}$ showed that the IMRT technique is safer for femurs than the 3D-CRT. Some authors recommend dose constraint for the femur to $b_{e} V_{50 G y}<5 \%$ [17]. However, in this study, these structures were not exposed to such high doses and therefore $\mathrm{V}_{40 \mathrm{~Gy}}$ was selected for analysis instead. The difference between the population average values of the $\mathrm{V}_{40 \text { Gy3D-CRT }}$ and $\mathrm{V}_{40 \text { GyIMRT }}$ $\left(\mathrm{V}_{\text {40Gy3D-CRT }}-\mathrm{V}_{\text {40GyIMRT }}\right)$ were $23.13 \%$ and $27.4 \%$ for the left and the right femur, respectively. These results demonstrate that the IMRT technique significantly reduces the volume of femurs receiving a dose of 40Gy and higher.

Another benefit of using the IMRT technique is improved sparing of the pelvic bones. The flat bones of the pelvis include red marrow which produces white blood cells, erythrocytes and platelets. The irradiation of the pelvic area, and thus of the pelvic bones can influence the hematopoietic function of red bone marrow. Mell et al. [18] report a decrease of haematological toxicity after reducing the dose delivered to the bone marrow for patients with rectal cancer. The ability to isolate the structure of bone marrow using PET for patients with gynaecological diseases was analysed by McGuire et al. [19]. The active bone marrow was regarded as an important critical organ. During irradiation of patients with a higher risk of nodal involvement, the pelvic bones were included in the treatment area. Analysis of 3D-CRT and IMRT plans demonstrates that average differences $\left(\mathrm{V}_{40 \mathrm{~Gy} 3 \mathrm{D}-\mathrm{CRT}}-\mathrm{V}_{40 \mathrm{GyIMRT}}\right)$ and $\left(\mathrm{V}_{30 \mathrm{~Gy} \text { 3D-CRT }}-\mathrm{V}_{\text {30GyIMRT }}\right)$ for pelvic bones were $8.02 \%$ and $9.15 \%$, respectively.

The QUANTEC recommends the following constrains: for the bladder $\left(\mathrm{V}_{65 \mathrm{~Gy}}<50 \%\right)$ and for the rectum $\left(\mathrm{V}_{50 \mathrm{~Gy}}<50 \%\right)$. 

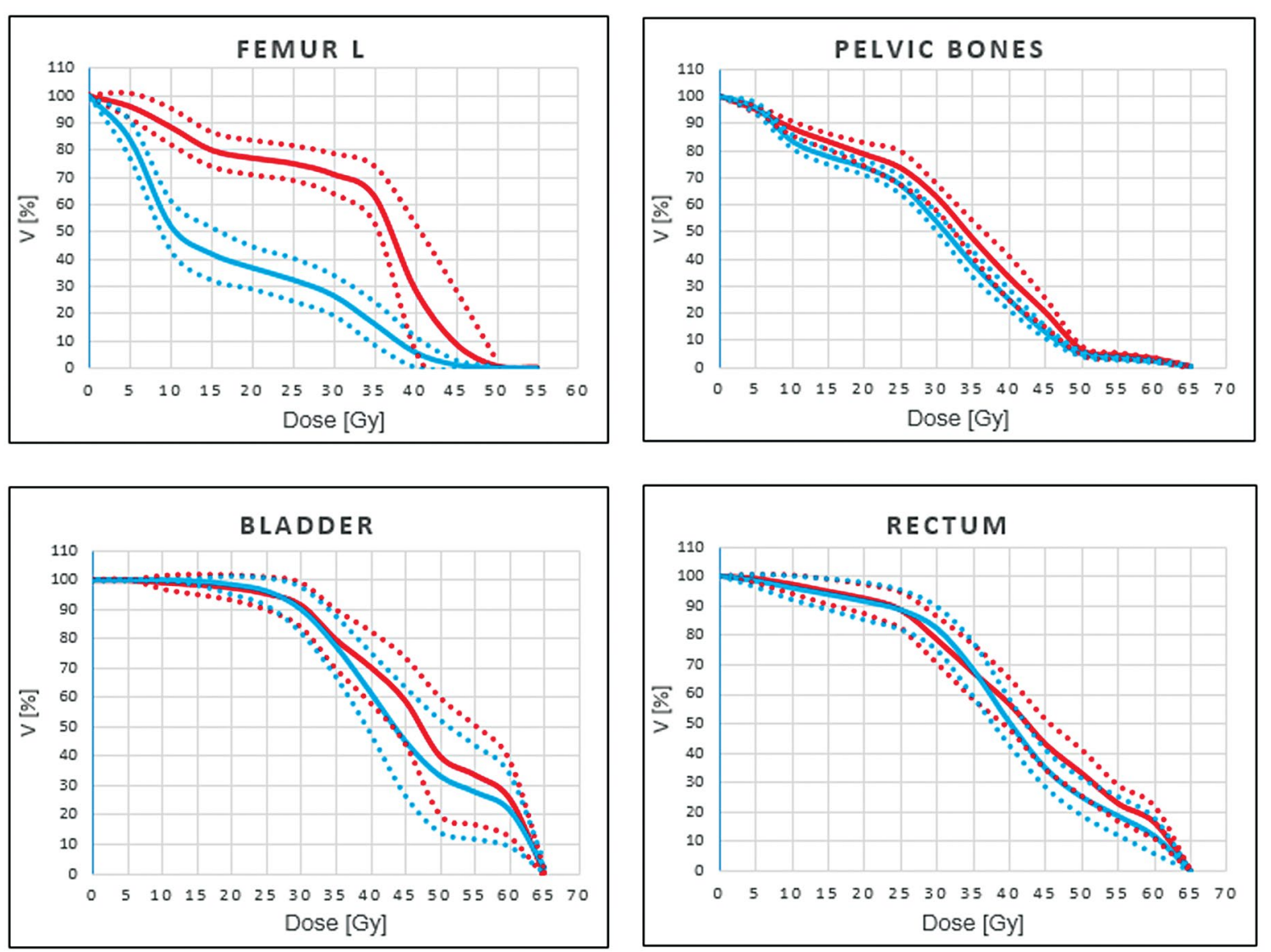

Figure 2. Average histograms of the OARs in 3D-CRT (red) and IMRT(blue) techniques. The dashed lines describe the average population cumulative dose volume histograms \pm standard deviation

Regardless of the technique, the doses received by the bladder and the rectum were significantly lower. However, better protection of the bladder and of the rectum was noticed in the 40-60 Gy dose range for IMRT plans.

According to the QUANTEC recommendations, the bowel bag value of $\mathrm{V}_{45 \mathrm{~Gy}}[\mathrm{cc}]$ was analysed. The Wilcoxon signed-rank test showed statistically significant differences in the bowel bag $\mathrm{V}_{45 \mathrm{~Gy}}[\mathrm{cc}$ ] between the techniques studied. These results are consistent with the work by Alongi et al. [14], which is based on clinical studies. Alongi noticed a reduction in toxicity to the bowel bag for the IMRT technique.

\section{Conclusions}

This study demonstrated that the IMRT technique for post-prostatectomy patients with a higher risk of nodal involvement is more advantageous than the 3D conformal technique. The IMRT technique resulted in better OAR sparing, especially for the pelvic bones. The dose distributions in all PTVs were similar to each other. The IMRT technique is more time consuming, due to the complexity of the planning process and of treatment verification. However, better sparing from the IMRT technique resulted in the introduction of the IMRT technique into clinical practice at our institute.

Maria Poncyljusz, M.Sc., C.S.

Maria Skłodowska-Curie Memorial Cancer Centre and Institute of Oncology, Warszawa, Poland Roentgena 5, 02-781 Warszawa, Poland tel: +48225462775

e-mail:sekretariat@zfm.coi.pl

\section{Conflict of interest: none declared}

\section{References}

1. Min M, Chua B, Guttner $Y$ et al. Is "pelvic radiation disease" always the cause of bowel symptoms following prostate cancer intensitymodulated radiotherapy? Radiother Oncol 2014; 110: 278-283.

2. Sutani S, Ohashi T, Sakayori M et al. Comparison of genitourinary and gastrointestinal toxicity among four radiotherapy modalities for prostate cancer: Conventional radiotherapy, intensity-modulated radiotherapy, and permanent iodine- 125 implantation with or without external beam radiotherapy. Radiother Oncol 2015; 117: 270-276.

3. Yorozu A, Tanaka T, Eriguchi T et al. IMRT Made Less Toxic Than 3D Conformal Radiation Therapy in Combination With lodine-125 Brachytherapy for the Treatment of Prostate Cancer. Int J Radiat Oncol Biol Phys 2015; 93: E188.

4. Neill CJ. Dosimetric comparison of intensity-modulated solutions for intact prostate cancer. Med Dosim 2014; 39: 366-372. 
5. Pesznyak C, Pócza T, Bencsik B et al. Comparison of normal tissue dosimetry for 3D-CRT and IMRT techniques in prostate irradiation. Radiother Oncol 2014; 111: S186.

6. Mellon EA, Javedan K, Strom TJ et al. A dosimetric comparison of volumetric modulated arc therapy with step-and-shoot intensity modulated radiation therapy for prostate cancer. Practical Radiation Oncology 2015; 5: 11-15.

7. Albarran JV, da Silva RG, Payano S et al. Acute toxicity hypofractionatedIMRT vs standard radiotherapy in prostate cancer: comparative study. Radiother Oncol 2016; 119: S646.

8. Russo D, Leone A, Papaleo A et al. Toxicity evaluation in adjuvant hypofractionated IMRT versus conventional 3DCRT in prostate cancer. Radiother Oncol 2014; 111: S96-S97.

9. Wortel R, Incrocci L, Pos F et al. Image-guided IMRT reduces late toxicity compared to 3D-CRT for prostate cancer. Radiother Oncol 2016; 119: S346-S347.

10. Carter HE, Martin A, Schofield D et al. A decision model to estimate the cost-effectiveness of intensity modulated radiation therapy (IMRT) compared to three dimensional conformal radiation therapy (3DCRT) in patients receiving radiotherapy to the prostate bed. Radiother Oncol 2014; 112: 187-193.

11. Katayama S, StrieckerT, Kessel Ket al. Hypofractionated IMRT of the prostate bed after radical prostatectomy: acute toxicity in the PRIAMOS-1 Trial. Radiother Oncol 2014; 90: 926-933.

12. Riou $O$, Laliberte $B, A z r i a ~ D$ et al. Implementing intensity modulated radiotherapy to the prostate bed: dosimetric study and early clinical results. Med Dosim 2013; 38: 117-121.
13. Spiotto MT, Hancock SL, King CR. Radiotherapy after prostatectomy: improved biochemical relapse-free survival with whole pelvic compared with prostate bed only for high-risk patients. Int J Radiat Oncol Biol Phys 2007; 69: 54-61.

14. Alongi F, Fiorino $C$, Cozzarini $C$ et al. IMRT significantly reduces acute toxicity of whole-pelvis irradiation in patients treated with post-operative adjuvant or salvage radiotherapy after radical prostatectomy. Radiother Oncol 2009; 93: 207-212.

15. Gay MD, Barthold HJ, O'Meara EC. Male pelvis normal tissue. RTOG consensus contouring guidelines. (https://www.rtog.org/LinkClik. aspx?fileticket...tabid=354).

16. Kavanagh BD, Pan CC, Dawson LA et al. Radiation dose-volume effects in the stomach and small bowel. Int J Radiat Oncol Biol Phys 2010; 76 (3 Suppl): S101-S107.

17. Davidson $M$, Blake $S$, Batchelar D et al. Assessing the role of volumetric modulated arc therapy (VMAT) relative to IMRT and helical tomotherapy in management of localized, locally advanced, and post-operative prostate cancer. Int J Radiat Oncol Biol Phys 2011; 80: 1550-1558.

18. Mell LK, Schomas DA, Salama JK et al. Association between bone marrow dosimetric parameters and acute hematologic toxicity in anal cancer patient treated with concurrent chemotherapy and intensity-modulated radiotherapy. Int J Radiat Oncol Biol Phys 2008; 70: 1431-1437.

19. McGuire SM, Menda Y, Ponto LL et al. A methodology for incorporating functional bone marrow sparing in IMRT planning for pelvic radiation therapy. Radiother Oncol 2011; 99: 49-54. 